\title{
Sicherstellung der Gesundheitsversorgung auf dem Lande
}

\section{UWE LÜBKING}

Uwe Lübking ist

Beigeordneter für Recht, Soziales, Bildung und Sport im DStGB - Deutscher Städte- und Gemeindebund, Berlin

\begin{abstract}
Aus dem Sozialstaatsprinzip des Grundgesetzes folgt die Schaffung gleichwertiger Lebensverhältnisse im ganzen Land. Dazu gehören auch die ärztliche und pflegerischer Versorgung. Auch wenn Deutschland im internationalen Vergleich über ein leistungsfähiges Gesundheitssystem verfügt, gibt es gerade in ländlichen und strukturschwachen Räumen Versorgungslücken. Die Politik und die Akteure des Gesundheitswesens sind gefordert, eine gute medizinische und pflegerische Versorgung sicherzustellen. Ein Baustein dürfte der Abbau der Sektorengrenzen sein, weitere die Förderung der Telemedizin sowie die Delegation ärztlicher Leistungen.
\end{abstract}

\section{Medizinische Versorgung: Grundlage für gleichwertige Lebensverhältnisse}

Deutschland verfügt unstreitig über ein leistungsfähiges Gesundheitssystem. Laut Bundesärztekammer gab es Ende 2018 knapp 392.500 berufstätige Ärztinnen und Ärzte, 2 Prozent mehr als im Vorjahr. Rechnerisch kommt eine Ärztin oder ein Arzt auf 211 Bürger, 1990 waren es noch 355. Mehr Mediziner führen aber nicht automatisch zu mehr Arbeitsleistungen. Im niedergelassenen Bereich nimmt die Zahl der Praxen ab. Von 2007 bis 2017 ist jede siebte Praxis geschlossen worden. Auch sind die Mediziner in unserem Land ungleich verteilt. Die Folge ist ein $\mathrm{Ne}-$ beneinander von über- und unterversorgten Regionen. Betroffen sind vor allem strukturschwache ländliche Gebiete, aber auch Stadtteile größerer Städte mit einer schwierigen Sozialstruktur. Dies ist mit dem Grundsatz der Gleichwertigkeit der Lebensverhältnisse nicht vereinbar.
Gleichwertigkeit darf nicht mit Einheitlichkeit der Lebensverhältnisse gleichgesetzt werden. Im Grundgesetz taucht der Begriff gleichwertiger Lebensverhältnisse zwar in Artikel 72 Abs. 2 auf, doch handelt es sich dabei lediglich um eine vorrangig staatsorganisationsrechtliche Bestimmung, die die Abgrenzung der Kompetenzen von Bund und Ländern in der Gesetzgebung näher regelt. Ein genereller Auftrag, für gleichwertige Lebensverhältnisse zu sorgen, lässt sich daraus nicht herleiten. Unmissverständlich ist der Auftrag zur Schaffung gleichwertiger Lebensverhältnisse dagegen im Raumordnungsgesetz des Bundes verankert. Nach $\mathbb{2}$ ROG sind im „Gesamtraum der Bundesrepublik Deutschland und in seinen Teilräumen [...] ausgeglichene soziale, infrastrukturelle, wirtschaftliche, ökologische und kulturelle Verhältnisse anzustreben. [...] Diese Aufgaben sind gleichermaßen in Ballungsräumen wie in ländlichen Räumen, in strukturschwa- 
chen wie in strukturstarken Regionen zu erfüllen. [...]“. Auch das im Grundgesetz verankerte Sozialstaatsprinzip verlangt von Politik und Verwaltung, allen Bürgerinnen und Bürgern den Zugang zu elementaren, für eine menschenwürdige Existenz unabdingbaren Leistungen der Daseinsvorsorge zu ermöglichen. Wünschenswert wäre eine explizite Verankerung der Schaffung gleichwertiger Lebensverhältnisse als Staatsziel im Grundgesetz. Dadurch

\section{Wünschenswert wäre eine Verankerung der Schaffung gleichwertiger Lebensverhältnisse als Staatsziel im Grundgesetz.}

würde die staatspolitische Bedeutung dieser Zielstellung und die Verantwortung aller Politikbereiche unterstrichen mit der Folge, dass der Handlungszwang erhöht würde.

Dies würde auch die medizinische Versorgung betreffen. Ein wesentlicher Aspekt gleichwertiger Lebensverhältnisse ist der Zugang zu Leistungen der Daseinsvorsorge. Dabei sind regionale Ungleichheiten bis zu einem gewissen Grad in einem so großen Staatsgebiet wie dem deutschen zu akzeptieren, zumal es aus individueller Sicht gewisse Kompensationsmöglichkeiten gibt. Gleichwertigkeit ist dann gegeben, wenn eine Mindestversorgung mit Dienstleistungen auch unter Nutzung der digitalen Möglichkeiten ortsnah vorhanden oder mit vertretbarem Aufwand erreichbar sind. Die Frage, welche Bereiche der Daseinsvorsorge eine Mindestversorgung umfassen soll, ist ganz entscheidend davon abhängig, was für einzelne Bevölkerungsgruppen elementar zum Leben gehört. Unstreitig dürfte sein, dass die Nahversorgung (insbesondere die Versorgung mit den Gütern des täglichen Bedarfs) aber insbesondere auch die medizinische Versorgung und Pflege elementar für Leben und körperliche Unversehrtheit sind. Mobilität kann außerdem dazu beitragen, lokale Verschlechterungen z.B. in der Nahversorgung oder medizinischen Versorgung durch bessere Erreichbarkeit naher Zentren zumin- dest teilweise zu kompensieren. Die hausärztliche Versorgung, aber auch Kindertageseinrichtungen und Grundschulen sind möglichst flächendeckend in den Grundzentren vorzuhalten, die auch mit dem öffentlichen Personennahverkehr gut erreichbar sein müssen. Ein Mangel an Allgemeinmedizinerinnen und Allgemeinmedizinern wiegt deshalb schwer, weil der Hausarzt die Zentralfigur bei der Gesundheitsversorgung der Bevölkerung ist. Nach einem Beschluss des Deutschen Ärztetages sind „Hausärztinnen und Hausärzte (...) die zentralen Ansprechpartner für die akute Versorgung und die kontinuierliche, oft lebenslange Betreuung der Bürger bei allen gesundheitlichen Problemen." Sie sind die „Lotsen“ im Gesundheitswesen. Eine wohnortnahe Erreichbarkeit ist daher zwingend. Über die allgemeine Grundversorgung hinaus sind natürlich gerade die Menschen in kleineren Dörfern darauf angewiesen, auch die stärker spezialisierten Angebote erreichen zu können. Deswegen ist es entscheidend, spezielle Angebote des Gesundheitswesens (insbesondere Fachärzte, Krankenhäuser und Apotheken), in leistungsfähigen Klein- und Mittelstädten als zentralen Orten zu bündeln, was eine Anbindung mit öffentlichen Verkehrsmitteln erleichtert. Darüber hinaus sind verstärkt die Möglichkeiten der Digitalisierung zu nutzen. (Siehe Beirat für Raumentwicklung- Räume entwickeln - Heimat gestalten! 2019)

\section{Kommission Gleichwertige Lebensverhältnisse}

Der Abschlussbericht der Facharbeitsgruppe 5 zur sozialen Daseinsvorsorge im Rahmen der Kommission Gleichwertige Lebensverhältnisse weist zu Recht darauf hin, dass die Gesundheits- und Pflegepolitik sowie die Politik für ältere Menschen in den nächsten Jahren vor großen Herausforderungen stehen. Es müsse eine wohnortnahe und qualitativ hochwertige ambulante und stationäre medizinische und pflegerische Versorgung, gut ausgebaute und verlässliche Angebote der Altenpflege sowie im Rahmen der kommunalen Daseinsvorsorge Angebote der örtlichen Altenhilfe in allen Teilen unseres Landes gewährleistet werden. Besondere Herausforderungen seien die demografischen Entwicklungen, wie die Alterung der Bevölkerung und damit verbunden ein Anstieg der Pflegebedürftigen, eine spürbare $\mathrm{Ab}$ wanderung aus manchen Regionen, aber auch die Tatsache, dass in den nächsten Jahren viele ältere Ärzte/innen in den Ruhestand gehen werden. Eine Politik, die dem Ziel der Gleichwertigkeit der Lebensverhältnisse in allen Teilräumen Deutschlands verpflichtet ist, müsse deshalb die verschiedenen gesundheitspolitische Themenfelder zu einer Strategie zusammenführen, die den spezifischen Besonderheiten sowohl der ländlichen Räume als auch der urbanen Ballungsräume gerecht werde. Krankenhäuser, Haus- und Fachärzte/innen, Apotheken, Pflegeeinrichtungen, Heilmittelerbringer und Geburtshilfen müssen weiterhin flächendeckend und wohnortnah zur Verfügung stehen. Hierzu bedürfe es insbesondere flexibler und zielgenauer Versorgungsangebote, die eine regionsspezifische Betrachtungsweise und individuelle Teilgebietslösungen berücksichtigen.

Im Einzelnen weist die Facharbeitsgruppe, in der Vertreter der unterschiedlichen Bundesressorts, der Bundesländer und der kommunalen Spitzenverbände vertreten waren, auf folgendes hin:

- Gerade bei der Gesundheitsversorgung in ländlichen und strukturschwachen Gebieten werde es darauf ankommen, die Gesundheitsversorgung stärker über Sektorengrenzen hinweg zu organisieren und dabei Aspekte der regionalen Entwicklung wie Mobilität und Erreichbarkeit, digitale Vernetzung und die Stärkung ehrenamtlicher Angebote in einem Gesamtzusammenhang zu betrachten. Die bis 2020 vorzulegenden Vorschläge der 2018 eingerichteten Bund-Länder-Reformkommission "Sektorenübergreifende Versorgung“ komme daher gerade im Hinblick auf die Sicherung einer flächendeckenden Versorgung große Bedeutung zu. Die Reformkommission soll Vorschläge vorlegen, wie die Zusammenarbeit und Vernetzung der Versorgungsangebote im Gesundheitswesen ausgebaut und verstärkt werden kann. Dabei sollen Spielräume für eine stärker regionale Ausgestaltung ermöglicht werden. 
- Auch zukünftig müsse eine wohnortnahe hausärztliche Versorgung und eine patientennahe Facharztversorgung gewährleistet sein. Es sei u. a. Aufgabe der Kassenärztlichen Vereinigungen, dies durch zusätzliche Steuerungsinstrumente, etwa mittels einer kleinräumigeren Planung, sicherzustellen, was aufgrund der gesetzlichen Vorgaben bereits möglich ist.

- Die medizinische Versorgung müsse gemeinsam mit Pflege und örtlicher Altenhilfe sozialräumlich ausgerichtet und besser abgestimmt erbracht werden. Dies setzte voraus, an den Schnittstellen der einschlägigen Sozialgesetzbücher zu verbessern.

- Besondere Bedeutung habe insbesondere die Verbesserung der Notfallversorgung. Hier ist eine enge Verzahnung der Akteure erforderlich.

- Telemedizinische Anwendungen sollen helfen, sowohl Grenzen zwischen Versorgungssektoren als auch räumliche Entfernungen zu überwinden. Deshalb solle der Einsatz dieser Anwendungen ausgebaut und mit dem notwendigen rechtlichen und finanziellen Rahmen ausgestattet werden.

- Bei der ambulanten medizinischen Versorgung müssen mehr Anreize geschaffen werden, Angebote auch in unterversorgten Regionen $\mathrm{zu}$ entwickeln. Die Attraktivität der vertragsärztlichen Versorgung für junge Ärzte/innen, soll gesteigert werden. Kommunen könnten hierzu mit attraktiven Arbeits- und Lebensbedingungen für Ärzte/innen und ihre Familien einen wichtigen Beitrag leisten.

- Neben einer praxisnahen, an den Bedürfnissen der Patienten/innen orientierten Ausbildung sei es sinnvoll, einen Teil der ärztlichen Leistungen an medizinisches Fachpersonal zu delegieren, um Ärzte/innen vor allem in unterversorgten Gebieten zu entlasten. Entsprechende Modellprojekte sollten ausgebaut werden.

- Die Krankenhausplanung und die Rahmenbedingungen für die Krankenhausfinanzierung müssten eine qualitativ hochwertige, patientenund bedarfsgerechte Versorgung der Bevölkerung mit Krankenhausleistungen sicherstellen, die auch dem Wirtschaftlichkeitsgebot Rechnung trage.
Leider ist es bekanntermaßen nicht zu einem gemeinsamem Abschlussbericht der Kommission Gleichwertige Lebensverhältnisse gekommen, in dem sich die Akteure auf klare Schlussfolgerungen und Maßnahmen auch zur zukünftigen Sicherung der gesundheitlichen und pflegerischen Versorgung verständigen konnten. Auch in dem Beschluss der Bundesregierung zu den ersten Umsetzungsschritten der Kommissionsergebnisse taucht dieses Handlungsfelde nicht auf. In den Schlussfolgerungen zu dem Bericht der Facharbeitsgruppe stellen die der Kommission vorsitzenden Ministerinnen und Minister (BMI, BMFSFJ, BMEL) immerhin fest, dass gerade bei der Gesundheitsversorgung in ländlichen und strukturschwachen Gebieten es darauf ankomme, diese stärker über Sektorengrenzen hinweg zu

\section{Unter dem Gesichtspunkt einer stetig steigenden Lebenserwartung wird die Notwendigkeit einer flächendeckenden Grundversorgung noch bedeutender werden.}

organisieren und dabei regionale Aspekte wie Erreichbarkeit, digitale Vernetzung oder die Stärkung ehrenamtlicher Angebote in einem Gesamtzusammenhang zu betrachten. Hier sei eine enge Verzahnung der Akteure erforderlich. Konkret wird empfohlen:

- Der mit dem Terminservice- und Versorgungsgesetz (TSVG) im ambulanten ärztlichen Bereich eingeschlagene Weg einer flexiblen Bedarfsplanung, Angebotssteuerung und Anreizschaffung soll konsequent weiterverfolgt werden, um in allen Sektoren der Gesundheits- und Notfallversorgung auch zukünftig eine flächendeckende und wohnortnahe Versorgung sicherzustellen.

- Flexible und innovative Instrumente wie der Einsatz telemedizinischer Anwendungen, die Delegation von ärztlichen Leistungen an medizinisches Fachpersonal oder mobile Versorgungsangebote (wie die „rollende Praxis“) sollten ausgebaut und mit dem notwendigen rechtlichen und finanziellen Rahmen ausgestattet werden, um Grenzen sowohl zwischen Versorgungssektoren als auch räumliche Entfernungen zu überwinden.

- In den für die Bedürfnisse einer alternden Bevölkerung besonders wichtigen Bereichen der Krankenhausversorgung und Pflege müssen weiterhin - gerade in strukturschwachen Räumen - verstärkte Anstrengungen unternommen werden, um Bedarfsgerechtigkeit, Finanzierbarkeit und einem steigenden Fachkräftebedarf Rechnung zu tragen.

Das BMI hat zwischenzeitlich die Akteure des Gesundheitswesens und der Pflege zu einem Dialogforum zur Sicherstellung der Versorgung eingeladen. Oberstes Ziel im ambulanten ebenso wie im stationären medizinischen Bereich muss die Sicherung der flächendeckenden medizinischen Versorgung sein, ausgerichtet am Bedarf vor Ort. Dies bezieht sich im Bereich der Fachkräfte nicht allein auf Ärztinnen und Ärzte, sondern auch auf andere Berufsgruppen, die zur Sicherung der medizinischen und pflegerischen Versorgung notwendig sind. Gerade unter dem Gesichtspunkt einer stetig steigenden Lebenserwartung wird in Zukunft die Notwendigkeit einer flächendeckenden Grundversorgung noch bedeutender werden. Um diese sicherzustellen gibt es keinen Königsweg. Notwendig ist es, unterschiedliche Ansätze umzusetzen.

\section{Sicherstellungsauftrag erfüllen}

Auch zukünftig müssen eine wohnortnahe hausärztliche Versorgung und eine patientennahe Facharztversorgung gewährleistet sein. Die Kassenärztlichen Vereinigungen (KVen) sind verpflichtet, die ärztliche Versorgung sicherzustellen, also dafür zu sorgen, dass genügend Ärztinnen und Ärzte mit der durch die Kassen zur Verfügung gestellten Vergütung vorhanden sind ( $\$ 75$ SGB V). Die Kassenärztlichen Vereinigungen können dies durch zusätzliche Steuerungsinstrumente, etwa mittels einer kleinräumigeren Planung, sicherzustellen, was aufgrund der gesetzlichen Vorgaben bereits möglich ist. Die Kassenärztlichen Vereini- 
gungen haben in den einzelnen Bundesländern unterschiedliche Maßnahmen zur gleichmäßigen Versorgung ergriffen. Hierzu gehören z. B. die Förderung von Praxiseröffnungen in unterversorgten Gebieten oder die Vergabe von Stipendien an Studenten, die sich nach dem Studium in unterversorgten Gebieten niederlassen. Die Maßnahmen reichen aber nicht aus. Auch die Vergütung der hausärztlichen Tätigkeit und für Kinderärzte gehört auf den Prüfstand. Der Sachverständigenrat für die Begutachtung der Entwicklung im Gesundheitswesen hat bereits in seinem Gutachten 2014 einen „Landarztzuschlag“ für Hausärztinnen und Hausärzte empfohlen, die in einem unterversorgten oder von Unterversorgung bedrohten Planungsbereich praktizieren. Dies kann für die Versicherten kostenneutral durch Vergütungsumschichtungen innerhalb der Ärzteschaft finanziert werden und ist ein weiterer wichtiger Baustein für die Erhöhung der Attraktivität der Niederlassung im ländlichen Raum. Das Vergütungssystem im Bereich der hausärztlichen Versorgung muss insgesamt ansprechender und bürokratiearm ausgestaltet werden. Die Ärztinnen und Ärzte müssen die tatsächlich von ihnen erbrachten Leistungen vergütet bekommen. Es darf nicht sein, dass die Landärztin oder der Landarzt aufgrund der Deckelung des Budgets schlechter bezahlte oder sogar unentgeltliche Mehrarbeit leisten muss. Ohne zusätzliche Maßnahmen wird sich das Niederlassungsverhalten weiter zu Lasten der strukturschwachen Gebiete verschieben. So könnten die Niederlassungszuschüsse durch Kassenärztliche Vereinigungen und Länder erhöht werden. Eine weitere Möglichkeit wäre, wie in einzelnen KVRegionen in Mecklenburg-Vorpommern schon praktiziert, in unterversorgten Gebieten neben der Honorarverteilung auch die Mengenbegrenzung zumindest befristet aufzuheben.

\section{Versorgungsstrukturgesetz weiter umsetzen, Sicherstellung weiter deregulieren}

Seit 2012 hat der Gesetzgeber verschiedene Maßnahmen zur Sicherstellung der medizinischen Versorgung ergriffen:

Mit dem Versorgungsstrukturgesetz von 2012 hat der Gesetzgeber eine Reihe von Maßnahmen zur Sicherstellung der flächendeckenden medizinischen
Versorgung ergriffen. Dazu gehören die Abschaffung der Residenzpflicht für Ärztinnen und Ärzte, wonach diese bislang auch im Ort ihrer Praxis wohnen mussten, ebenso wie die einfachere $\mathrm{Zu}$ lassung von Zweitpraxen. Vertragsärzte und Medizinische Versorgungszentren können Ärztinnen und Ärzte anstellen, wenn für die Fachrichtungen keine $\mathrm{Zu}-$ lassungsbeschränkungen bestehen, also in unterversorgten Gebieten. Hier können auch Teilzeitmodelle für Ärztinnen und Ärzte zur Vereinbarkeit von Familie und Beruf angeboten werden.

Darüber hinaus sollen die Kassenärztlichen Vereinigungen von der Möglichkeit Gebrauch machen, in unterversorgten Gebieten eigene Praxen zu betreiben.

Das GKV-Versorgungsstärkungsgesetz von 2015 sieht weitere Schritte vor: Kassenärztliche Vereinigungen können einen sog. Strukturfond zur Finanzierung von Fördermaßnahmen zur Sicherung der ärztlichen Versorgung einrichten. Die Gründungsmöglichkeiten für Medizinische Versorgungszentren (MVZ) wurden weiterentwickelt. Kommunen insbesondere in strukturschwachen Regionen können die Gründung eines MVZ aktiv mitgestalten oder auch selbst gründen. Mit dem Innovationsfond können zukunftsweisende Versorgungsformen gefördert werden. Ziel ist es, die Regelversorgung durch eine bessere Verzahnung der Versorgung durch niedergelassene Ärztinnen und Ärzte und Krankenhäuser sowie die verstärkte Zusammenarbeit unterschiedlicher Facharztrichtungen und Berufsgruppen weiter zu entwickeln.

Das Terminservice- und Versorgungsgesetz von 2019 normiert einen für die KVen künftig obligatorisch Strukturfonds zur Sicherung der Versorgung vor allem in ländlichen Gebieten. Die KV kann Eigeneinrichtungen betreiben oder telemedizinische Angebote aufbauen, um die Versorgung sicher-zustellen. Bei festgestellter Unterversorgung muss die KV innerhalb von 12 Monaten eine Eigeneinrichtung betreiben oder telemedizinische Angebote aufbauen. Auch bei der Organisation von Medizinischen Versorgungszentren (MVZ) gibt es Veränderungen. So dürfen anerkannte Praxisnetze künftig Versorgungszentren gründen, und zwar unabhängig von drohender oder festgestellter Unterversorgung.

Hausärzte müssen wohnortnah zur Verfügung stehen. Die Aufstellung eines Bedarfsplans dient der Sicherstellung der vertragsärztlichen Versorgung. Der Bedarfsplan stellt den Stand und den Bedarf an ärztlicher Versorgung dar. Nach der BedarfsplanungsRichtlinie des Gemeinsamen Bundesausschusses ist Planungsbereich für die hausärztliche Versorgung der Mittelbereich in der Abgrenzung des Bundesinstituts für Bau, Stadt und Raumforschung (BBSR). Laut der aktuellen Definition des BBSR bilden diese Mittelbereiche die Verflechtungsbereiche für die Versorgung mit Gütern des gehobenen Bedarfs ab und sind in der Regel einem Mittelzentrum oder einem mittelzentralen Verbund beziehungsweise höherrangigen zentralen Ort zugeordnet. Diese sogenannten Hausärztlichen Planungsbereiche sind vielfach schon allein aufgrund ihrer flächenmäßigen Größe nicht geeignet, den tatsächlichen Bedarf und den tatsächlichen Stand der hausärztlichen Ver-

\section{Die Überarbeitung der Bedarfsplanungsrichtlinie sollte zum Anlass genommen werden, künftig auf Grundzentren abzustellen.}

sorgung abzubilden. Gerade mit Blick auf die zentrale Stellung der Hausärztin oder des Hausarztes im Gesundheitswesen ist eine Erreichbarkeit des Arztes am Wohnort unverzichtbar. Es nützt den Menschen überhaupt nichts, wenn die hausärztliche Versorgung in einem großen Planungsbereich insgesamt sichergestellt werden kann, aber es vor Ort keine Hausärztin oder keinen Hausarzt mehr gibt und die Mobilität fehlt. Hier bedarf es einer kleinräumigeren Steuerung, die eine Umverteilung von Ballungen im Mittelzentrum in die Grundzentren vorsieht. Deshalb sollte die aktuelle Überarbeitung der Bedarfsplanungsrichtlinie auf Bundesebene zum Anlass genommen werden, künftig auf die Grundzentren abzustellen, also derjenigen zentralen Orte in den Gemeinden, an denen die Einrichtungen für die Versorgung der Bürger mit den Dingen des täglichen Be- 
darfs (Grundversorgung) konzentriert werden sollen. Begleitend dazu sollten Hausarztsitze raumordnungsrechtlich an den Grundzentren festgesetzt werden. Im Kern muss also die Bedarfsplanung im hausärztlichen Bereich „heruntergezont" werden.

Die Bundesländer haben eigene Maßnahmen zur Sicherstellung der ärztlichen Versorgung in strukturschwachen Gebieten ergriffen, z. B. in Form von Niederlassungszuschüssen. Die Länder können auch die Bildung von Medizinischen Versorgungszentren in Form von Ärztegenossenschaften unterstützen.

\section{Gemeindliche Aktivitäten}

Die Städte und Gemeinden haben ein hohes Interesse daran, dass sich vor Ort ausreichend Medizinerinnen und Mediziner zur Versorgung der Einwohnerinnen und Einwohner niederlassen. Sie unterstützen die ärztliche Versorgung im Rahmen ihrer Zuständigkeiten. Kommunen sollten bei der Ärztewerbung ein ähnliches Standortmarketing betreiben wie bei der Ansiedlung von Betrieben und Unternehmen. Dazu gehört z.B., einen frühzeitigen Aktionsplan bei drohender Schließung einer Arztpraxis mit den Kassenärztlichen Vereinigungen zu entwerfen. Dies kann und sollte in enger Kooperation mit den umliegenden Kommunen und dem Landkreis erfolgen. Einzelne Kommunen haben darüber hinaus u.a. folgende Maßnahmen ergriffen: Stipendien für Studenten mit späterer Niederlassungsverpflichtung, Förderung von medizinischen Praktika im Ort, Ankauf von geeigneten Immobilien für eine Praxis, Darlehen für den Betrieb, Unterstützung bei der Arbeitsplatzsuche für Familienangehörige der Ärztin oder des Arztes, Hilfe bei der Kinderbetreuung, Ermöglichung von Teilzeitarbeit, Kooperationen zur schnellen Verankerung des neuen Arztes in der Gemeinde. Ärztinnen und Ärzte werden sich nämlich nur dann in einer Gemeinde ansiedeln, wenn diese ein attraktiver Lebensort ist. Von daher kommt der Umsetzung der Ergebnisse der Kommission Gleichwertige Lebensverhältnisse zur Stärkung strukturschwacher Kommunen besondere Bedeutung zu.

Eine schleichende Übertragung des Sicherstellungsauftrags auf die Kommunen darf damit aber nicht verbun- den sein. Dies gilt auch für den Betrieb einer eigenen ärztlichen Einrichtung. Dies würde viele Städte und Gemeinden hinsichtlich finanzieller und Haftungsfragen überfordern. Es darf auch nicht sein, dass sich die Akteure im Gesundheitswesen darauf verlassen, dass „wohlhabende“ Städte und Gemeinden sich Hausärzte durch z.B. finanzielle Unterstützung leisten können, andere aber nicht.

\section{Mobilitätskonzepte weiterentwickeln}

Die Erreichbarkeit der Arztpraxen spielt gerade bei einer älter werdenden Gesellschaft eine immer größere Rolle. Notwendig ist es, Mobilität und Erreichbarkeit in die Versorgungsplanung mit einzubeziehen. Damit ein Zugang der Versicherten zur ambulanten Versorgung gewährleistet werden kann, sollten die zuständigen Aufgabenträger beim Ausbau und der Ausgestaltung des Öffentlichen Personennahverkehrs stets die Niederlassungssitze der Ärztinnen und Ärzte sowie die Öffnungszeiten der Praxen im Blick haben. Flankierend dazu sind Konzepte zu entwickeln, wie die Patientinnen und Patientinnen zum Arzt gelangen können. Darüber hinaus sind Mobilitätskonzepte zu entwickeln, die insbesondere auch gehbehinderten Patienten die Möglichkeit bieten, die Praxen aufzusuchen. Beispiele sind Bürgerbusse, Landarzt- oder Ruftaxen.

\section{Landarztquote}

Der Masterplan Medizinstudium 2020 ermöglicht den Ländern die Einführung einer sogenannten Landarztquote. Die Länder können danach bis zu 10 Prozent der Medizinstudienplätze vorab an Bewerberinnen und Bewerber vergeben, die sich verpflichten, nach Abschluss des Studiums und der fachärztlichen Weiterbildung in der Allgemeinmedizin für bis zu zehn Jahre in der hausärztlichen Versorgung in unterversorgten bzw. durch Unterversorgung bedrohten ländlichen Regionen tätig zu sein. Einige Bundesländer haben bereits von dieser Möglichkeit Gebrauch gemacht. Notwendig wären darüber hinaus die Stärkung und der Ausbau der Institute für Allgemeinmedizin. Für eine bedarfsgerechte und flächendeckende Versorgung der Bürgerinnen und Bürger ist es unerlässlich, weitere Studentinnen und Studenten für das Fach Allgemeinmedizin zu gewinnen. Das derzeitige Verhältnis in der Ausbildung der Allgemein- und Fachmedizin kann die fehlenden Hausarztplätze nicht auffüllen. Medizinstudentinnen und -studenten sollten sehr frühzeitig in hausärztlichen Praxen hospitieren und einen Einblick in die allgemeinmedizinische Versorgung erhalten. Patenschafts- und Mentoringprogramme, die dazu dienen, Hemmnisse bei der Niederlassung abzubauen, sind weiter auszubauen. Mit Blick auf den großen Bedarf an Hausärztinnen und Hausärzten muss die erst in den vergangenen Jahren mehr und mehr erfolgte Institutionalisierung der Fachrichtung der Allgemeinmedizin in den medizinischen Fakultäten weiter forciert werden.

\section{Möglichkeiten zur Gewinnung von Ärztinnen und Ärzten verbessern}

Die Anwerbung ausländischer Ärztinnen und Ärzte oder auch die Vergabe von Stipendien an Studenten, die sich nach dem Studium in unterversorgten Regionen als Hausarzt niederlassen, sind bislang wenig erfolgreich. Das Anerkennungsverfahren für ausländische Mediziner sind zu kompliziert und dauern zu lange. Dies muss unbedingt vereinfacht werden, um tatsächlich Ärztinnen und Ärzte aus dem Ausland gewinnen zu können. Es bleibt abzuwarten, ob durch das Fachkräfteeinwanderungsgesetz positive Effekte eintreten.

\section{Krankenhäuser einbeziehen}

Die Krankenhäuser leisten schon heute einen wesentlichen Beitrag zur ambulanten Versorgung der Bevölkerung. Die ambulante Notfallversorgung wäre ohne die Krankenhäuser selbst in den Ballungsgebieten kaum noch zu gewährleisten. Vor dem Hintergrund des zunehmenden Ärztemangels im ambulanten Bereich kommt gerade den ländlichen Krankenhäusern eine besondere Bedeutung in der wohnortnahen Grund- und Regelversorgung zu. Diese Grund- und Regelversorgungskrankenhäuser müssen finanziell in die Lage versetzt werden, ihren Daseinsvorsorgeauftrag zu erfüllen und die medizinische Versorgung der Bevölkerung dauerhaft sicherzustellen. 
Notwendig ist, dass die Leistungen der Grundversorgung im Abrechnungssystem einen entsprechenden Stellenwert erhalten, der Anreize bietet, diese Aktivitäten zu verstärken.

Eine Studie der Bertelsmann-Stiftung, nach der die Zahl der Kliniken in Deutschland auf weniger als 600 verringert werden sollte, hat zu einer heftigen Diskussion gesorgt. Die Studie wird der Versorgungsrealität nicht gerecht. Auch ist die Behauptung fraglich, dass die Patienten immer einen ausgewiesenen Spezialisten brauchen. In einer älter werdenden Gesellschaft werden Krankenhäuser zunehmend auch für „einfache“ Erkrankungen wie Diabetes oder Nierenproblemen aufgesucht. Die Corona Erkrankungen zeigen auch, dass ausreichend Bettenkapazitäten für

\section{In den strukturschwachen ländlichen Räumen geht es auch um das Gefühl, nicht weiter abgehängt zu werden.}

Notfälle zur Verfügung stehen müssen.

Eine wohnortnahe Grundversorgung, eine zeitnahe Notfallversorgung und Spitzenmedizin in den Häusern der Maximalversorgung müssten gleichzeitig gewährleistet bleiben. Richtig ist, dass es in einzelnen Regionen, insbesondere in Ballungsräumen, eine Überversorgung gibt. Auf der anderen Seite mangelt es in Regionen bereits heute nicht nur an stationären Angeboten, sondern auch an niedergelassenen Ärzten. Die Kassenärztlichen Vereinigungen haben die Versorgungsengpässe im ambulanten Bereich seit Jahren nicht lösen können. Im ländlichen Raum geht es bei der Diskussion um die Schließung von Krankenhäusern aber auch um eine ganz andere Frage. Hier sorgt ein Krankenhaus in der Nähe nicht nur für eine gefühlte Sicherheit im Krankheitsfall. In den strukturschwachen ländlichen Räumen geht es auch um das Gefühl, nicht weiter abgehängt zu werden.

Es geht bei den Krankenhäusern im ländlichen Raum nicht zuletzt aber auch um die medizinische Versorgung für die Mehrzahl aller Patienten, die keine medizinische Maximalbehandlung benötigen. Bei einem Großteil der Versorgung in den Krankenhäusern handelt es sich nicht zuletzt wegen der fehlenden niedergelassenen Ärzte um medizinische Grundversorgung, insbesondere der altersbedingten Krankheitsbilder der Inneren Medizin. Das sind Behandlungen, die möglichst wohnortnah in erreichbaren Krankenhäusern auch in Zukunft erbracht werden müssen. Wer als Patient aufgrund seiner Krankheit den Spezialisten braucht, wird eine Spezialklinik aufsuchen. Für diese Krankheiten macht aus Sicht der Qualitätssicherung eine Zentralisierung Sinn, denn hier sind Spezialisten gefordert.

Man braucht also zum einen eine Krankenhausplanung, die die regionalen Besonderheiten in den Blick nimmt und Parallelstrukturen abbaut, zum anderen aber dringend eine bessere Verzahnung von ambulanter und stationärer medizinischer Versorgung einschließlich der Notfallversorgung. Bei einer älter werdenden Gesellschaft muss auch die Pflege mitgedacht werden. Andere Länder haben nämlich nicht nur ihre Krankenhausstruktur verändert, wie es die Studie als einzi-

Therapeuten und Pflegediensten. Der Neubau umfasst neben einer modernen Bereitschaftspraxis auch Untersuchungsund Behandlungsräume für verschiedene Fachdisziplinen. Die medizinisch notwendigen Versorgungen sollen auf sich ändernde Bedarfe ausgerichtet und aus einer Hand ambulant und stationär erbracht werden. Termine, Therapien oder Notfallmaßnahmen werden von zentraler Stelle koordiniert.

\section{Sektorengrenzen überwinden}

Die Versorgung der Bevölkerung kann zukünftig nur sichergestellt werden, wenn die Kooperation und Vernetzung der verschiedenen Fachdisziplinen und Sektoren wie Hausärzte, Fachärzte und Krankenhäuser gelingt. Die durch die Trennung von ambulanten und stationären bedingten Doppelstrukturen insbesondere im Facharztbereich müssen miteinander verknüpft werden. Eine BundLänder Arbeitsgruppe soll bis 2020 Vorschläge für die Entwicklung einer solchen sektorenübergreifende Versorgung erarbeiten. Wünschenswert wäre, wenn die Planung und Sicherstellung der medizinischen Versorgung zukünftig regional und durch die Selbstverwaltungspartner unter Einbeziehung der Kommunen gemeinsam gelöst werden könnte.

Präventionsprogrammen sowie dem grundlegenden Ausbau der ambulanten Versorgung flankiert.

Wer auch immer mit welchen Ideen den Krankenhaussektor verändern will, muss dem grundgesetzlichen Auftrag der Sicherstellung der Daseinsvorsorge und dem Gebot der Gleichwertigkeit der Lebensverhältnisse Rechnung tragen. Wer Krankenhäuser schließen möchte, muss die gesamte medizinische Versorgungsrealität einbeziehen. Patienten müssten flächendeckend auf eine wohnortnahe Gesundheitsversorgung zugreifen können. Vor allem muss die Frage beantwortet werden, wie man der zunehmenden Behandlungs- und Pflegebedürftigkeit in der Gesellschaft bei gleichzeitigem Fachkräftemangel begegnen will.

Statt der Schließung von Krankenhäusern können diese in ambulant-stationäre Zentren umgewandelt werden. In Brandenburg ist mit Mitteln des Innovationfonds ein Krankenhaus entsprechend umgebaut worden: Das Konzept setzt auf eine enge Vernetzung von Haus- und Fachärzten, Krankenhaus, Apotheken,

\section{Gesundheitszentren etablieren}

Gerade in ländlichen Regionen sind Gemeinschaftspraxen, Ärztehäuser oder lokale Gesundheitszentren dahingehend weiterzuentwickeln, dass Hausärzte und Fach-ärzte, medizinische Fachangestellte oder Arztassistenten und Pflegekräfte gemeinsam Leistungen anbieten. Der Sachverständigenrat hatte in seinem Gutachten bereits 2014 entsprechende Gesundheitszentren in ländlichen Regionen gefordert. Dort könnten z. B. auch angestellte Ärztinnen und Ärzte aus der Stadt tageweise arbeiten. Die Gesundheitszentren könnten mit den Kliniken und Pflegeeinrichtungen für die älter werdende Gesellschaft integrierte Versorgungskonzepte anbieten. Notwendig sind allerdings neue gemeinsame Finanzierungswege durch Krankenkassen, Pflegekassen, Kassenärztliche Vereinigungen sowie weitere Institutionen im Gesundheitswesen. 


\section{Substitution und Delegation - Berufsbild der Arztassistentin / des Arztassistenten einführen}

Wenn Ärztinnen und Ärzte eine zunehmend knappe Ressource werden, müssen Modelle gefördert werden, mit denen die vorhandenen medizinischen Kapazitäten optimaler genutzt werden können. So sind die Delegation und Substitution ärztlicher Leistungen weiter zu fördern. Unter den Kürzeln VERAH (Versorgungsassistentin in der Hausarztpraxis), MoNi (Medizinische Fachangestellte im Modell „Niedersachsen“), MoPra (Mobile Praxisassistentin), AGnEs (Arztentlastende gemeindenahe E-Health gestützte systemische Intervention) gibt es in einzelnen Regionen verschiedene Ausprägungen dieser Delegationsmodelle.

Denkbar wäre auch, dass die entsprechend ausgebildeten Kräfte in einer verwaisten ländlichen Arztpraxis einen stationären Anlaufpunkt für Patienten bekommen, indem sie selbst kleinere Krankheiten nach entsprechender Ausund Fortbildung behandeln können. Bei komplizierteren Fällen können sie dann Haus- oder Fachärzte hinzuziehen, die qualifiziert entscheiden, ob der Patient vor Ort versorgt werden kann oder in eine Fahrpraxis oder Klinik überwiesen wird.

\section{Es sollte auch überlegt werden, ob ein neues Berufsbild des Arztassistenten oder der Arztassistentin eingeführt wird.}

\section{Telemedizin etablieren, Chancen der Digitalisierung nutzen}

Zur Unterstützung der fachärztlichen Versorgung rücken auch telemedizinische Ansätze immer mehr ins Blickfeld. Sie stellen eine sinnvolle und notwendige Ergänzung der hausärztlichen Versorgung im ländlichen Raum dar. Durch die vermehrte Nutzung von Telemedizin kann die präklinische gesundheitliche Versorgung insbesondere in ländlichen Gebieten mit langen Fahrtwegen grundsätzlich optimiert und die Versorgungsqualität bei der Erstversorgung der Patienten verbessert werden.

Damit Telemedizin tatsächlich vermehrt als Unterstützung eingesetzt werden kann, muss die Nutzung verstärkt ermöglicht werden. Dies bezieht sich zum einen auf die Technik und Fragen des Breitbandausbaus, zum anderen auch auf berufsrechtliche Grundlagen. Die elektronische Patientenakte muss schnellst möglichst eingeführt werden. Voraussetzung ist zwingend, dass der Breitbandausbau in Deutschland weiter vorangetrieben und ein flächendeckendes Hoch-

Auch sollte überlegt werden, ob ein neues Berufsbild des Arztassistenten oder der Arztassistentin eingeführt wird. Dies würde sich bei der ärztlichen Versorgung entlastend auswirken, ohne dass die Qualität der Versorgung beeinträchtigt wird. KBV und Bundesärztekammer unterstützen aktiv das Berufsbild des Physician Assistants, der solche Aufgaben übernehmen könnte. Das BMG sollte die erforderlichen Schritte zur Ausgestaltung der rechtlichen und finanziellen Rahmenbedingungen zur Durchführung von Modellvorhaben nach $\mathbb{} 63$ SGB V auf den Weg bringen, insbesondere die Anforderungen an diese Modellvorhaben leistungsgeschwindigkeitsnetz etabliert wird. Es müssen die noch bestehenden Vorbehalte über die Fernbehandlung ausgeräumt werden. In der Schweiz können Patienten per Telefon beraten werden. Die Mehrheit der Patienten kann durch Befragung und durch Blick auf Fotos behandelt werden. Der Rest wird an Praxen und Krankenhäuser verwiesen. In Baden-Württemberg soll es zumindest Modellversuche geben.

Die Telemedizin hat allerdings nur dann eine Chance, wenn die Leistungen angemessen vergütet und Bestandteil der Regelversorgung werden. Das Versorgungsstrukturgesetz sieht vor, dass eine Einigung darüber erzielt werden soll, welche telemedizinischen Leistungen in die Regelversorgung der gesetzlichen Krankenversicherung aufgenommen werden. Dieser Ansatz muss zügig umgesetzt werden. Die Coronakrise führt zu einem "Schwung" bei der Digitalisierung. Viele Ärzte entdecken nun die Vorzüge der digitalen Sprechstunde, was auch damit zu tun hat, das diese neuerdings komplett honoriert wird. Auch können die KVen entsprechende Angebote fördern und sind nach dem Terminservice- und Versorgungsgesetz hierzu auch bei einer Unterversorgung verpflichtet. Ein Beispiel ist der „Medibus" in Nordhessen, der im Auftrag der KV Hessen fünf Gemeinden bedient. Der von der DB Regio vermietete Bus bietet eine moderne Arztpraxis und den Einsatz von Telemedizin, um einen Fachkollegen per Videokonferenz zu Rate zu ziehen. Diese Medibusse könnten ein Beispiel für andere unterversorgte Regionen sein. Die KVen sind aufgerufen, derartige Modelle zu fördern.

Das Land NRW plant ein virtuelles Krankenhaus, in dem Patienten über Telemedizin behandelt werden können. Es soll keinen persönlichen Arztbesuch ersetzen, sondern eine Ergänzung bieten. Wenn beispielsweise in einer Praxis eine spezielle Expertise fehlt, können Hausärzte das virtuelle Krankenhaus kontaktieren. Gleichzeitig soll es aber auch ermöglichen, dass Patienten aus Regionen mit fehlenden Krankenhäusern und Fachärzten ebenfalls per Telemedizin von dem virtuellen Krankenhaus behandelt werden.

\section{Notärztliche Versorgung}

Immer mehr Menschen suchen die Notaufnahmen der Krankenhäuser und die Notambulanzen auf. Die Gründe sind vielschichtig: die Patienten glauben, in den Notaufnahmen schneller behandelt zu werden, die Öffnungszeiten der Praxen stehen mit dem Lebens- und Arbeitsrhythmus der Patienten nicht in Einklang, die Notambulanzen haben am Wochenende und Abend geöffnet, chronisch Kranke versprechen sich dort eine bessere Versorgung und Zuwanderer aus dem Ausland kennen die Struktur der niedergelassenen Ärzte nicht.

Der Bundesgesundheitsminister strebt eine Reform der Notfallversorgung mit 
dem Ziel an, die ambulante, stationäre und rettungsdienstliche Notfallversorgung zu einem System der integrierten Notfallversorgung zusammenzuführen. Die Kassenärztlichen Vereinigungen und die Krankenhäuser sollen künftig gemeinsam an ausgewählten Kliniken „Integrierte Notfallzentren“ betreiben, die als erste Anlaufstelle für Notfallpatienten dienen. Sie sollen jederzeit zugänglich sein und entscheiden, ob die Patienten an den Rettungsdienst, in die Krankenhausambulanz, die Bereitschaftsdienstpraxis oder eine Arztpraxis weitergeleitet werden. Der Gedanke, die Strukturen der Notfallversorgung der Kassenärztlichen Vereinigungen an den Krankenhäusern räumlich anzugliedern, ist grundsätzlich richtig. Hierbei sollen kleinere Krankenhäuser der Grundversorgung weiterhin Aufgaben der Notfallversorgung übernehmen dürfen. Abzulehnen sind Vorstellungen, die Bereitschaftsdienste mit den Rettungsleitstellen zusammenzuführen. Die kommunalen Rettungsdienste haben sich bewährt und funktionieren. Vielmehr ist der ärztliche Bereitschaftsdienst der Krankenkassen vielerorts nicht ausreichend ausgestattet. Notwendig wäre es, die Patienten von vornherein an die richtige Stelle zu leiten. Darüber hinaus müssen die „Portal- oder Bereitschaftspraxen", also Behandlungsräume, die die KVen unmittelbar am Krankenhaus unterhalten und finanzieren, ausgebaut werden. Auch muss das Luftrettungssystem weiterentwickelt und ausgebaut werden.

\section{Pflege einbeziehen}

Die medizinische Versorgung muss gemeinsam mit der Pflege- und Altenhilfe sozialräumlich ausgerichtet und besser abgestimmt werden. Die setzt voraus, dass bestehende Grenzen in den einschlägigen Sozialgesetzbüchern überwunden und die Verantwortung der Kommunen bei der Steuerung gestärkt werden. Daher ist ein grundlegender sozialer Wandel unerlässlich. Es ist dringend notwendig, sämtliche Ressourcen und Potentiale der verschiedenen gesellschaftlichen Akteure zu bündeln und zu vernetzen. Ziel ist das flexible Zusammenwirken von generationenübergreifenden Wohn- und Betreuungsformen, Nachbarschaftshilfe, professioneller Medizin, Pflege und Rehabilitation, technischer Assistenz und zivilgesell- schaftlich getragenen Teilhabemöglichkeiten im öffentlichen Raum. Die Städte und Gemeinden müssen die soziale Quartiersentwicklung als Pflichtaufgabe wahrnehmen können und die hierfür erforderliche Finanzausstattung erhalten. Geeignet wäre ein Bund-Länder- Programm „Daseinsvorsorge“, aus dem die Kommunen ausgestattet werden könnten. Der soziale Austausch der Quartiersbewohner und die Stärkung von sozialem Engagement und Mitverantwortung im Quartier können durch Begegnungsmöglichkeiten und Nachbarschaftsbeziehungen gestärkt werden. Für bedarfsgerechte, individuelle und flexible soziale Angebote im Quartier müssen Leistungserbringer ihre professionellen Dienstleistungen stärker differenzieren. Dafür ist ein flexibleres Sozialleistungsrecht eine unerlässliche Voraussetzung. Die Leistungsansprüche sollten künftig nicht mehr in unflexiblen Leistungskategorien, sondern als flexibel einsetzbare Budgetleistungen gewährt werden. Um passgenaue Unterstützungsarrangements im Sozialraum verwirklichen zu können, müssen die starren Abgrenzungen zwischen Säulen und Sektoren überwunden werden. Die Hospiz- und Palliativversorgung muss einbezogen und ausgebaut werden.

\section{Fazit}

Deutschland kann im internationalen Vergleich zwar auf eine qualitativ hochwertige Gesundheitsversorgung mit in der Regel gutem Zugang zu wohnortnahen Gesundheitsdienstleistungen blicken. Auf der anderen Seite gibt es insbesondere in strukturschwachen ländlichen Regionen zunehmend Probleme beim Zugang zu notwendigen Gesundheitsdienstleistungen. Dies steht im Widerspruch zum aus dem Sozialstaatsprinzip folgenden Grundsatz der Gleichwertigkeit der Lebensverhältnisse. Der Gesetzgeber ist daher verpflichtet, die notwendigen Maßnahmen zu ergreifen, damit für alle Menschen unabhängig von ihrem Wohnort eine wohnortnahe medizinische und pflegerische Versorgung zur Verfügung steht. Dies gilt auch, wenn bestehende Sicherstellungsaufträge nicht erfüllt werden. Eine gleichwertige Gesundheitsversorgung heißt aber auch schnelles und leistungsfähiges Breitband und Mobilfunk sowie eine moderne Verkehrsinfrastruktur.
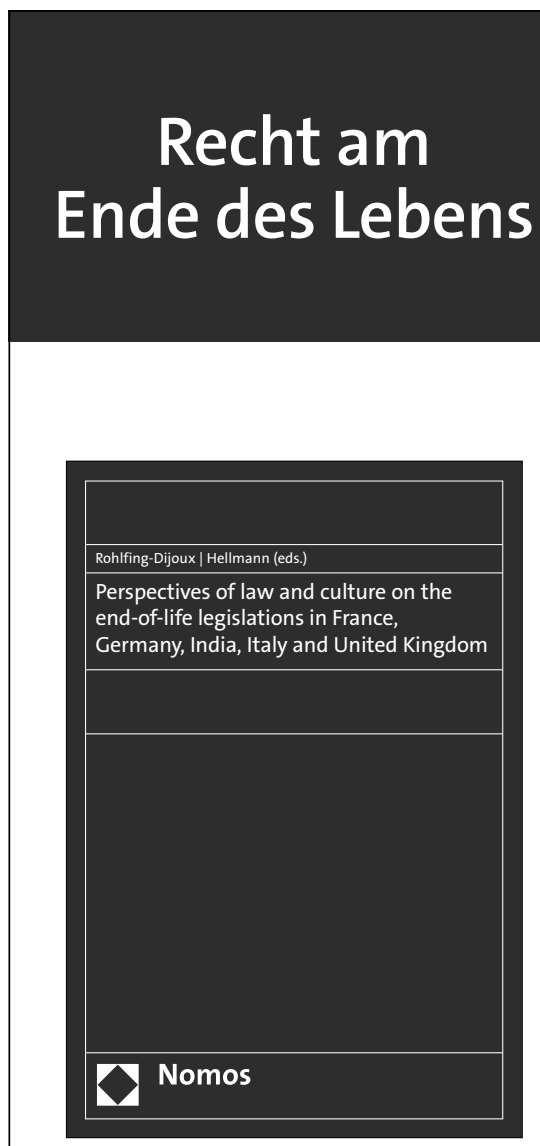

Perspectives of law and culture on the end-of-life legislations in France, Germany, India, Italy and United Kingdom

Herausgegeben von Prof. Dr. Stephanie Rohlfing-Dijoux und Prof. Dr. Uwe Hellmann

2019, 311 S., brosch., 79,- $€$ ISBN 978-3-8487-5492-2

In englischer Sprache

Das Buch widmet sich den rechtlichen Fragen, die durch die Problematik des Lebensendes aufgeworfen werden. Die Beiträge verbinden den Rechtsvergleich zwischen Frankreich, Deutschland, Italien, Indien und Großbritannien mit einer Pluridisziplinarität zwischen Recht, Medizin, Philosophie und Ethik.

\section{三 e Library}

X Nomos 